\title{
INTERPRETATION OF SOURCE COUNTS AND REDSHIFT DATA IN EVOLUTIONARY UNIVERSES
}

J. V. Wall, T. J. Pearson and M. S. Longair

Mullard Radio Astronomy Observatory,

Cavendish Laboratory, Cambridge, England

Conventional interpretation of the $\mathrm{N}(\mathrm{S})$ relation requires cosmic evolution of the radio source population. Investigators agree on the general features of this evolution: it must be confined to the most luminous sources, and must be strong, the numbers of such sources at redshifts of 1 to 4 exceeding the present numbers by a factor $\geq 10^{3}$. There is no consensus as to whether density or luminosity evolution prevails (or both), whether a cutoff in redshift is necessary, or whether the source populations found in high-frequency surveys follow even the general evolutionary picture deduced for the low-frequency survey population. It is therefore hardly surprising that the physical basis of the evolution, the ultimate goal of $N(S)$ interpretation, remains largely "in the realm of imaginative speculation" (P. A. G. Schever).

Recently the observational basis for source count interpretation has been greatly strengthened. There now exist $N(S)$ relations at frequencies of $408,1410,2700$ and $5000 \mathrm{MHz}$ which all reach to $210^{5}$ sources $\mathrm{sr}^{-1}$, and for which the statistical definition at $\mathrm{S}<1$ Jy is greatly improved over that available for previous investigations. Furthermore, at high flux densities at all frequencies, identifications and redshifts approach completeness and therefore luminosity distributions may be defined with greater certainty. We have undertaken a numerical investigation of the implications of this much-improved data base for cosmic histories of the different radio source populations. The early results are reported here.

\section{A SCHEME FOR SOURCE COUNT ANALYSIS}

We wish to determine $\rho(P, z$, type), the generalized luminosity function, which describes the spatial density of radio sources of luminosity $P$ and given type at all redshifts $z$. The function may be factored as $\rho=F\left(P, z\right.$, type). $\rho_{0}$ (P, type); $F$ is the evolution function and $\rho_{0}$ is the local luminosity function. 
We have developed a new numerical scheme which guarantees that these models will at least be consistent with luminosity distributions defined at high flux densities. Consider a single type of source, and suppose that for these sources a complete luminosity distribution $n(P)$ for $\mathrm{S}>\mathrm{S}_{0}$ is available. For a given world model and evolution function $\mathrm{F}(\mathrm{P}, \mathrm{z})$, the local luminosity function may be obtained directly:

$$
\rho_{0}(\mathrm{P}) \mathrm{dP}=\mathrm{n}(\mathrm{P}) \mathrm{dP} / \int_{0}^{z\left(\mathrm{~S}_{0}\right)} \mathrm{F}(\mathrm{P}, \mathrm{z}) \mathrm{dV}(\mathrm{z})
$$

where $z\left(S_{0}\right)$ is the redshift at which a source of luminosity $P$ has an observed flux density $\mathrm{S}_{0}$. The count for this type of source is then computed in the usual manner:

$$
\mathrm{N}(>\mathrm{S})=\int_{0}^{\infty} \mathrm{dP} \int_{0}^{z(S)} \mathrm{F} \cdot \rho_{0} \cdot \mathrm{dV}
$$

A complete luminosity distribution, statistically adequate in size, therefore makes it possible to proceed directly to a predicted count for each postulated evolution function $F$. The requisite input guesses for our approach are thus (i) a world model and (ii) an evolution function, while input data supplied are (iii) a luminosity distribution $\mathrm{n}\left(\mathrm{P}, \mathrm{S}_{0}\right)$ and (iv) an observed source count $\mathrm{N}(\mathrm{S})$; the procedure is to use (i), (ii) and (iii) to calculate the model count directly as above, and to compare it statistically via a $x^{2}$-test with (iv), the observed $\mathrm{N}(\mathrm{S})$ for this type of object. At the outset we have used simple analytic forms of evolution functions, whose free parameters are allowed to vary widely. The evolution models which survive the $x^{2}$-test may be examined in more detail by comparison with observational data at lower flux densities.

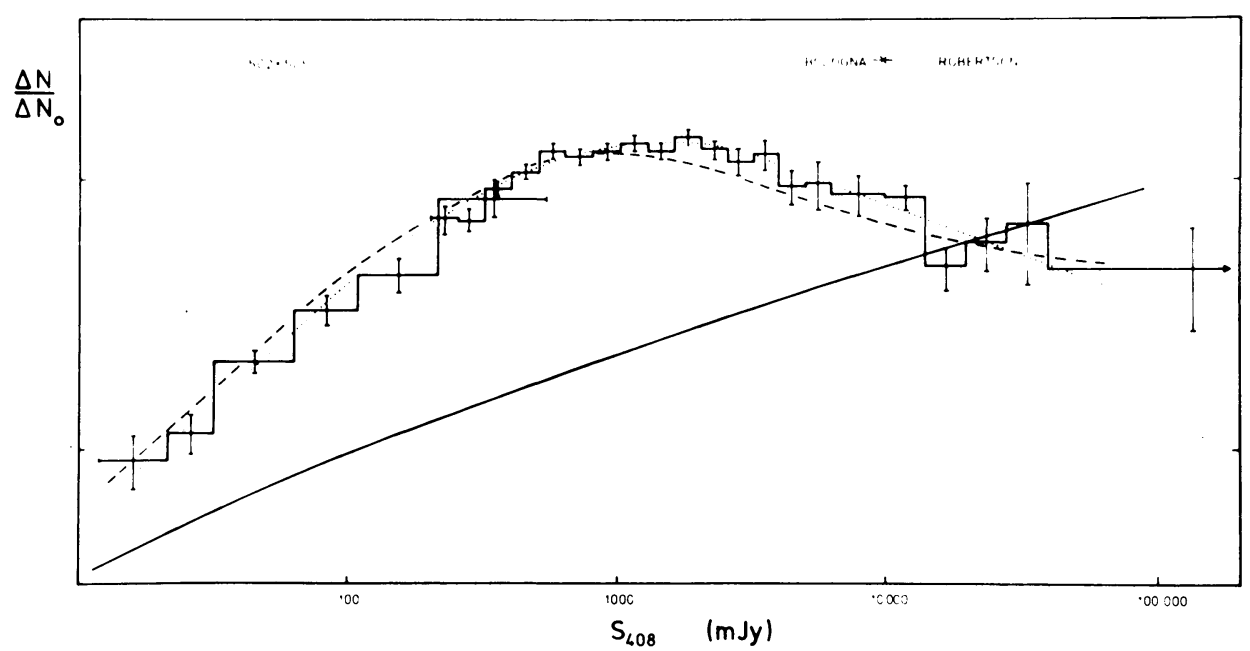

Figure 1. The $408 \mathrm{MHz}$ source count. Solid curve: model 1; dashed: model 2a (optimized); dotted: model 5 (optimized). 


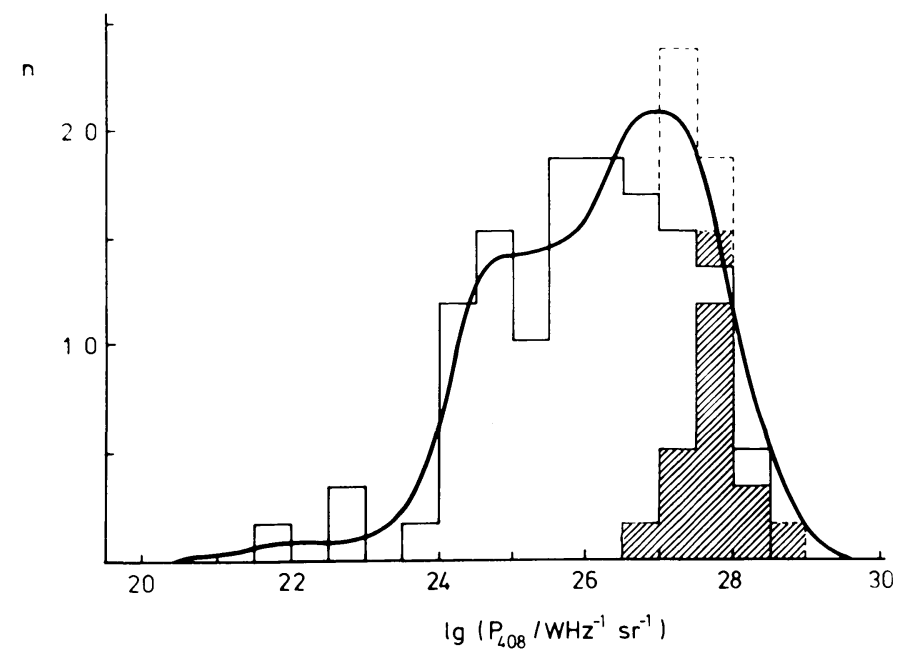

Figure 2. The $408 \mathrm{MHz}$ luminosity distribution, $\mathrm{S}_{408}>10 \mathrm{Jy}, 5.86 \mathrm{sr}$. Hatched area: QSOs; dotted area: no redshift estimates available; solid curve derived by smoothing the unbinned data.

\section{THE $408 \mathrm{MHz}$ SOURCE COUNT}

The evolutionary requirements of the $408 \mathrm{MHz}$ count (Fig. 1) are particularly amenable to exploration by the present method. Some $90 \%$ of all sources found at this frequency are of the extended, steepspectrum type, so that a valid first approximation is to assume that the count consists of only these sources, with a spectral index $\alpha=0.75$ $\left(S \propto v^{-\alpha}\right)$. Moreover, recent identifications and redshift determinations yield the wel1-defined luminosity distribution of Fig. 2, the details of which will be described elsewhere.

The guesses of our initial investigation are (i) world models of Friedmann geometries with $\Omega=0$ and $1, \mathrm{H}_{0}=50 \mathrm{~km} \mathrm{~s}^{-1} \mathrm{Mpc}^{-1}$, and (ii) the following forms for the evolution function $F$.

1. $F(P, z)=1$ (non-evolutionary, source-conserving)

2. $F(P, z)=X_{1}(P)+\phi X_{2}(P)$, with $\phi=\exp \left\{M\left(1-t / t_{0}\right)\right\}$, and $\mathrm{x}_{1}=\left(\mathrm{P}_{\mathrm{c}} / \mathrm{P}\right)^{\mathrm{m}} /\left\{1+\left(\mathrm{P}_{\mathrm{c}} / \mathrm{P}\right)^{\mathrm{m}}\right\}, \mathrm{x}_{2}=1 /\left\{1+\left(\mathrm{P}_{\mathrm{c}} / \mathrm{P}\right)^{\mathrm{m}}\right\}$

2a: no redshift cutoff; $2 \mathrm{~b}: \mathrm{F}=0$ if $z>z_{\mathrm{c}}$.

3. As for $2 b$, but with $\phi(P, z)=(1+z)^{\beta}$ for $z<z_{c}$.

4. $F(P, z)=\exp \left\{M(P)\left(1-t / t_{0}\right)\right\}$, with

$$
\begin{aligned}
& M(P)=0 \text { for } P<P_{1}, M(P)=M_{\max } \text { for } P>P_{2} \text {, and } \\
& M(P)=M_{\max }\left(\operatorname{lgP}-\lg P_{1}\right) /\left(1 g P_{2}-\lg P_{1}\right) \text { for } P_{1}<P<P_{2} ;
\end{aligned}
$$


4a: no redshift cutoff; $4 \mathrm{~b}: \mathrm{F}=0$ if $z>\mathrm{z}_{\mathrm{C}}$.

5. As for $2 a$, but with $\operatorname{lgP}_{c}=a \operatorname{lgz}+b$.

The first three are conventional models from the literature. Al1 fail to produce satisfactory models for the $408 \mathrm{MHz}$ source count for

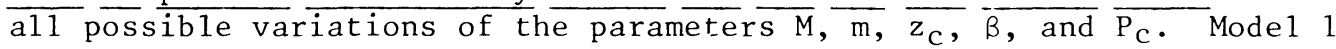
(no evolution) produces a count which differs from that observed to an extreme degree of statistical significance, as is evident in Fig. 1 . Model $2 \mathrm{a}$ has attractively few parameters ( $\left.\mathrm{M}, \mathrm{P}_{\mathrm{C}}, \mathrm{m}\right)$, but after optimizing these, is rejected at the $99.9 \%$ level of significance. The difficulty (Fig. 1) is in obtaining the rapid turnover towards the faint flux densities when enough evolution is supplied to fit the steeply-rising count of bright flux densities; and when this turnover is achieved, falloff is too rapid. Imposing a redshift cutoff (2b) aggravates this behaviour, and model 3 is even worse in this respect because the density enhancement increases rapidly up to the redshift cutoff. The basic problem is that these models produce too many sources at the low-luminosity end of the evolving component. Models 4 and 5 were invented to avoid the difficulty. Model 4 was derived by examining how the evolution function depends on radio luminosity; the value of $\left\langle\mathrm{V} / \mathrm{V}_{\mathrm{m}}\right\rangle$ as a function of luminosity for sources with $\mathrm{S}_{408}>5$ Jy was used to define $M(P)$, and in this sense there are no free parameters in $4 \mathrm{a}$, and only one $\left(z_{c}\right)$ in $4 \mathrm{~b}$. However, we succeeded in finding acceptable models of type 4 only in the $\Omega=1$ geometry. Model 5 has the transition luminosity between evolving and non-evolving components as a function of redshift, thereby reducing the contribution to the count of weak, high-redshift sources. It produces the best fit to the source count, and bears considerable resemblance to luminosity evolution. The dependence of luminosity function on epoch given by models 4 and 5 is shown in Fig. 3, and the optimum parameters are as follows :

$$
\begin{aligned}
& \text { Mode1 4a: } \operatorname{lg~} \mathrm{P}_{1}\left(\mathrm{~W} \mathrm{~Hz}^{-1} \mathrm{sr}^{-1}\right)=26.0,1 \mathrm{~g} \mathrm{P}_{2}=27.1, \mathrm{M}_{\max }=11.5 \\
& \text { 4b: } \lg \mathrm{P}_{1}=25.0,1 \mathrm{~g} \mathrm{P}_{2}=27.3, \mathrm{M}_{\max }=11.0, \mathrm{z}_{\mathrm{C}}=3.5 \\
& \text { 5: } \quad \mathrm{M}=9.8, \mathrm{a}=3.14, \mathrm{~b}=26.8, \mathrm{~m}=1.06 \text {. }
\end{aligned}
$$

These models are very different in character, as exemplified by the redshift distribution which each predicts at low flux densities (Fig. 4); the identification content of such a sample clearly provides a powerful discriminant. For example, the identifications of Richter (1975) for the 5 C 3 survey suggest to us that $z 35 \%$ of all sources with $\mathrm{S}_{408}>10 \mathrm{mJy}$ have redshifts less than 0.6 . The proportions of sources with $z<0.6$ are $26 \%$ and $42 \%$ for mode $1 \mathrm{~s} 4 \mathrm{a}$ and $4 \mathrm{~b}$, and $28 \%$ for model 5 , and it is therefore not clear that models $4 \mathrm{a}$ and 5 remain tenable. It is clear that identifications and redshifts for even a small sample of sources at this level would provide powerful constraints to the permitted forms of evolution. 

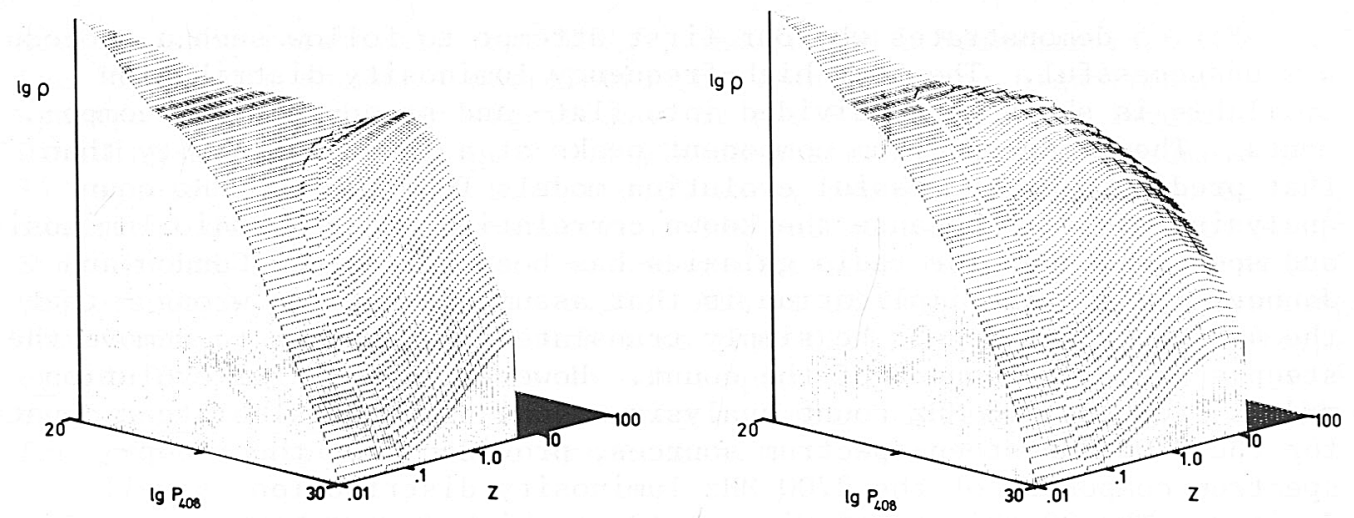

Figure 3. Dependence of luminosity function on redshift in model $4 \mathrm{~b}$ (left) and model 5 (right).

\section{THE $2700 \mathrm{MHz}$ SOURCE COUNT}

In surveys at frequencies above $408 \mathrm{MHz}$, the proportion of compact objects with 'flat' radio spectra becomes significant and increases as survey frequency increases. Consider for simplicity that two classes of source are represented in the $2700 \mathrm{MHz}$ count: 'flat-spectrum' and 'steep-spectrum' sources. A first attempt to derive evolution models for these two populations might proceed as follows. (1) Assume that the steep-spectrum objects are the same as those in the $408 \mathrm{MHz}$ count. (2) Translate a successful model for the $408 \mathrm{MHz}$ count to $2700 \mathrm{MHz}$, obtaining the appropriate normalization either from the spectral index distribution at some $S_{2700}$ or from the 'steep-spectrum' component of the $2700 \mathrm{MHz}$ count (Fig. 5 of Wall, this volume). (3) Subtract this from the total $2700 \mathrm{MHz}$ count to obtain a complete count for the flatspectrum sources. (4) Analyse this with the procedure of section 1 to set limits on the evolution required for these sources.
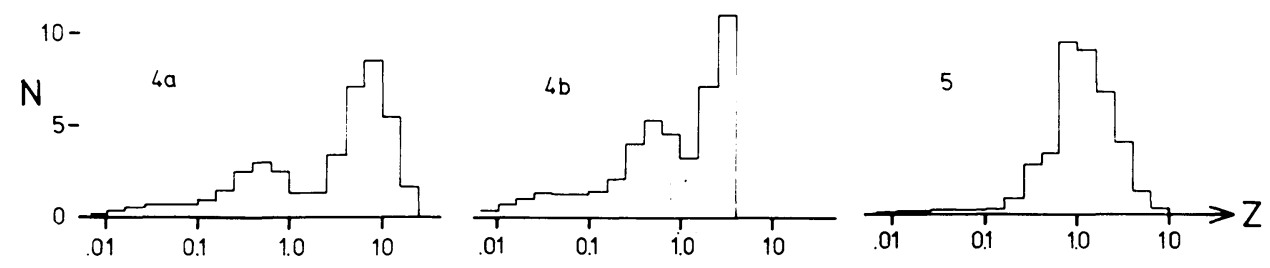

Figure 4. Predicted distributions of redshifts for sources with $10<\mathrm{S}_{408}<50 \mathrm{mJy}$; models $4 \mathrm{a}, 4 \mathrm{~b}$ and 5 . 
Fig. 5 demonstrates why our first attempt to follow such a procedure was unsuccessful. The best high-frequency luminosity distribution available is shown here, divided into flat- and steep-spectrum components. The steep-spectrum component peaks at a lower luminosity than that predicted by successful evolution models from the $408 \mathrm{MHz}$ count analysis. This is because the known correlation between radio luminosity and spectral index for radio galaxies has been neglected (Condon and Jauncey 1974). The implication is that assumption (1) is wrong - that the $408 \mathrm{MHz}$ count cannot be simply translated to $2700 \mathrm{MHz}$ to remove the steep-spectrum component of the count. However, successful evolution models from the $408 \mathrm{MHz}$ count analysis may be used to calculate a count for the $2700 \mathrm{MHz}$ steep-spectrum sources, provided that the steepspectrum component of the $2700 \mathrm{MHz}$ luminosity distribution is well defined. The 28 objects of Fig. 5, 12 of which do not have measured redshifts, are clearly inadequate in this regard. Moreover, the flatspectrum component of the luminosity function contains only 20 objects, and exploration of the evolution function for flat-spectrum sources on the basis of such a sample is somewhat optimistic.

There is nevertheless some indication that the cosmic history of the flat-spectrum population differs considerably from that of the steep-spectrum population. There are differences amongst the source counts themselves (Figures 4 and 5 of Wall, this volume). These may be due to differences in luminosity functions rather than evolution functions, but there is further indication that this is not so. C. J. Masson has measured magnitudes to $\pm 0^{\mathrm{m}} \cdot 3$ for a complete sample of 55 QSOs in unobscured regions of the $\pm 4^{\circ}$ declination zone of the Parkes $2700 \mathrm{MHz}$ survey. Redshifts for many of these were kindly communicated to us by R. Lynds and $\mathrm{D}$. Wills, and the $\left\langle\mathrm{V} / \mathrm{V}_{\mathrm{m}}\right\rangle$ for the sample (Masson and Wall, in preparation) is $0.58 \pm 0.03$, significantly less than the values of 0.65 to 0.7 obtained for objects from low-frequency surveys (Schmidt, this volume). The result suggests that flat-spectrum

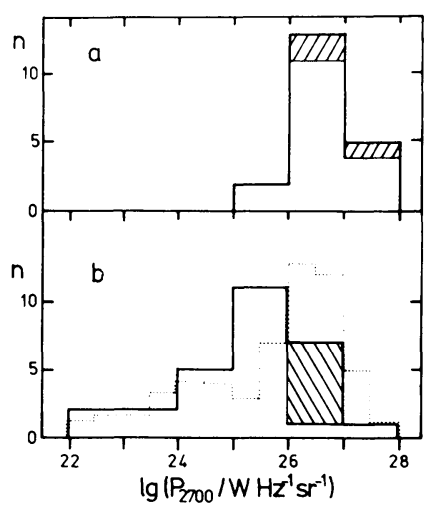

Figure 5. The $2700 \mathrm{MHz}$ luminosity distribution (a) for flat-spectrum sources, (b) for steep-spectrum sources. Hatched areas: no redshift estimates available; dotted histogram: prediction from $408 \mathrm{MHz}$ results. 
sources show relatively low $\left\langle\mathrm{V} / \mathrm{V}_{\mathrm{m}}\right\rangle$, and indeed a Spearman rank test on the 55 objects showed a correlation between $\mathrm{V} / \mathrm{V}_{\mathrm{m}}$ and high-frequency spectral index which is significant at the $95 \%$ confidence level.

Dividing the sample at $\alpha_{\mathrm{HF}}=0.5$ yields a steep-spectrum subsample of 14 objects with $\left\langle\mathrm{V} / \mathrm{V}_{\mathrm{m}}\right\rangle=0.70 \pm 0.05$ whose properties appear to be identical with the (steep-spectrum) 3CR and 4C samples studied by Schmidt and by Lynds and Wills, and a flat-spectrum subsample of 41 objects with $\left\langle\mathrm{V} / \mathrm{V}_{\mathrm{m}}\right\rangle=0.54 \pm 0.04$. A11 41 of these QSOs have structures dominated by compact components less than $0 " .1$ arc in extent (Bentley et a1. 1976). These results do not require a complete lack of evolution for compact QSOs, but they do imply different cosmic histories for QSOs of different radio structures.

\section{CONCLUSIONS}

Some authors have argued that satisfactory evolution functions are trivial to obtain, firstly because of a wide range of permissible assumptions - geometry, local luminosity function, and of course the form of the evolution itself, and secondly because of highly permissive data - poorly defined source counts, luminosity distributions, background temperature requirements. In the face of modern data this point of view is hard to sustain, and the definition of satisfactory models becomes distinctly non-trivial. Progress is therefore possible on investigating the form of the evolution function for different source populations, and in particular on providing constraints on this evolution. From our examination of the $408 \mathrm{MHz}$ counts we conclude that all conventional models of evolution for steep-spectrum sources are unsatisfactory, and that identifications and redshifts of sources at the flux density level of the $5 \mathrm{C}$ surveys provide powerful constraints on models which do result in correct source counts. From our preliminary $2700 \mathrm{MHz}$ results we conclude that existing luminosity distributions permit only the crudest of analyses, but the difference in evolution for compact and and extended QSOs suggested by the $<\mathrm{V} / \mathrm{V}_{\mathrm{m}}>$ test provides considerable incentive to obtain the necessary observations for this investigation.

Bentley, M., Haves, P., Spencer, R.E. and Stannard, D.: 1976, Month1y Notices Roy. Astron. Soc. 176, 275.

Condon, J.J. and Jauncey, D.L.: 1974, Astron. J. 79, 1220 .

Richter, G.A.: 1975, Astron. Nachr. 296, 65.

\section{DISCUSSION}

Murdoch: Does the luminosity evolution force a sharp modification to the density law in the vicinity of $z=3$ ?

Wal2: We have considered basically density evolution; of our successful models, type 1 works (in $q_{0}=0.5$ geometry) both with (la) and without 
(1b) redshift cut-offs, while type 2 imposes an effective redshift cutoff by progressively narrowing the range of luminosities allowed to evolve.

Murdoch: Robertson (Sydney) is doing similar work, but instead of parametrized models he uses an array of numbers for $\delta(z)$ which are modified until convergence to the source counts is obtained. The conclusions are similar: $(1+z) \beta$ must be modified, and in particular it is forced to drop sharply at $z \sim 3$.

Wal2: Robertson's approach is one that we are very interested in trying. The difficulty comes in communicating the answers; if you have to invent a parameterized model to describe the resultant array, you might as well have started with this model in the first place. It has always been known that $(1+z)^{\beta}$ models require a redshift cut-off.

Grueff: You stressed the necessity of optical identifications at the $5 \mathrm{C}$ flux density level. Did you compare the predictions of your most successful models with the data already available at $\sim 1$ Jy? The 1 Jy point in the $\log \mathrm{N}-\log \mathrm{S}$ is a rather interesting one, since it is there that we observe the largest source excess with respect to Euclidean prediction.

Wal2: I confess not; our look at the identification data has been preliminary, and we went for the longest baseline in flux density, namely to what is known of the $5 \mathrm{C}$ identifications. Our luminosity distribution is "defined" at $10 \mathrm{Jy}$; $1 \mathrm{Jy}$ is not very far away considering the breadth of the luminosity function.

E.M. Burbidge: I am still puzzled as to how you estimate redshifts for radiogalaxies which do not have measured redshifts.

Wall: We do what we can from estimated magnitudes and the $\mathrm{m}-\mathrm{z}$ plot for radio galaxies. But I want to emphasize that extreme assumptions about these objects do not affect the structure of the luminosity distribution markedly, and in particular do not change the conclusions of the analysis.

Roeder: Why did your (1/2) model not work in the empty universe, $q_{0}=0$ ?

Wall: The difficulty is at the faint (5C) end of the $408 \mathrm{MHz}$ count, and is the same as the one which destroys the "conventional" models of the literature. The model in question just fits the counts with $q_{0}=0.5$ geometry, because the relatively small volumes, $\Delta \mathrm{V}$, corresponding to the $\Delta z$ 's assist in converging the faint source counts gradually; these $\Delta V^{\prime}$ 's are much larger for $q_{0}=0$, and the faint counts take on quite the wrong shape.

Ekers: We know from the bivariate luminosity function analysis presented by Perola that there is a variation of optical luminosity for the lower radio power ranges, so the estimation of aisiance for these galaxies could be affected. Perhaps you should consider including the bivariate 
luminosity function in your analysis so that you can correctly include the galaxies with no redshift determination.

Wal2: I agree in principle. However, from several considerations, it is clear that the missing-z objects have high radio powers, off the top of the well-defined part of Perola's bivariate function, and before this refinement becomes necessary, the missing redshifts will probably be available. 